\title{
Prevalence of anxiety and its correlates among older adults in Latin America, India and China: cross-cultural study
}

\author{
A. Matthew Prina, Cleusa P. Ferri, Mariella Guerra, Carol Brayne and Martin Prince
}

\section{Background}

Anxiety is a common mental disorder among older people who live in the Western world, yet little is known about its prevalence in low- and middle-income countries.

\section{Aims}

We investigated the prevalence of anxiety and its correlates among older adults in low- and middle-income countries with diverse cultures.

\section{Method}

Cross-sectional surveys of all residents aged 65 or over $(n=15021)$ in 11 catchment sites in 7 countries (China, India, Cuba, Dominican Republic, Venezuela, Mexico and Peru) were carried out as part of the 10/66 collaboration. Anxiety was measured by using the Geriatric Mental State Examination (GMS) and the Automated Geriatric Examination for Computer Assisted Taxonomy (AGECAT) diagnostic algorithm.

\section{Results}

The age- and gender-standardised prevalence of anxiety varied greatly across sites, ranging from $0.1 \%(95 \% \mathrm{Cl} 0.0$ 0.3) in rural China to $9.6 \%(95 \% \mathrm{Cl} 6.2-13.1)$ in urban Peru. Urban centres had higher estimates of anxiety than their rural counterparts with adjusted (age, gender and site) odds ratios of 2.9 (95\% Cl 1.7-5.3). Age, gender, socioeconomic status and comorbid physical illnesses were all associated with a GMS/AGECAT diagnosis of anxiety, and so was disability (World Health Organization Disability Assessment Schedule II).

\section{Conclusions}

Anxiety is common in Latin America. Estimates from this region are similar to the ones from high-income European countries found in the literature. As demographic change will occur more rapidly in these countries, further research exploring the mental health of older people in developing areas is vital, with the inclusion of other specific anxiety disorders, along with evidence for strategies for supporting those with these disorders.

\section{Declaration of interest}

The 10/66 Dementia Research Group works closely with Alzheimer's Disease International, the non-profit federation of 77 Alzheimer associations around the world. Alzheimer's Disease International is supported in part by grants from GlaxoSmithKline, Novartis, Lundbeck, Pfizer and Eisai.
Little is known about the epidemiology of anxiety among older adults living in low- and middle-income countries. This disorder, which can substantially impair quality of life ${ }^{1}$ and has also been associated with increased risk of mortality ${ }^{2,3}$ and disability, ${ }^{4}$ is thought to decrease with age ${ }^{5}$ but it still common among older people living in high-income countries. ${ }^{6}$ It is not known whether similar patterns occur across the world. A recent systematic review of anxiety ${ }^{6}$ in later life only reported studies conducted in high-income countries, and we are aware of only a handful of studies that estimated the prevalence of anxiety outside those settings. ${ }^{7-10}$ The $10 / 66$ study provides an opportunity to estimate anxiety in diverse settings but using a standardised and validated methodology. We also aimed to investigate sociodemographic factors and health statuses associated with both clinical and subclinical anxiety and the impact that this condition has on disability. We anticipated finding a lower prevalence of anxiety in low- and middle-income countries but expected it to be as disabling as it is in high-income countries.

\section{Method}

\section{Study design}

Cross-sectional surveys of all residents aged 65 or over $(n=$ 15021 ) in 11 catchment areas in 7 countries (China, India, Cuba, Dominican Republic, Venezuela, Mexico and Peru) were carried out as part of the baseline phase of the 10/66 Dementia Research Group's population-based programme. The full protocol has been published elsewhere. ${ }^{11}$ Catchment area sites were selected purposively with a view to identifying typical central urban high density, and predominately low socioeconomic status districts in national or state capital cities (Beijing in China, Chennai in India, Havana and neighbouring Matanzas in Cuba, Santo Domingo in Dominican Republic, Caracas in Venezuela, Mexico City in Mexico and Lima in Peru) and contrasting rural areas, with a traditional agrarian lifestyle and low-density population in the four countries where this was feasible (villages around Vellore in India, Daxing in China, Morelos state in Mexico, and Canete in Peru). The boundaries of each catchment area were precisely defined, and households mapped. Each household was then systematically door-knocked to identify all those aged 65 years and over, who were then considered eligible for participation in the survey. The response rate was excellent in most centres and ranged from 72 to $98 \%$ (average $86 \%$ ). After the consent was taken the full assessment, lasting $2-3 \mathrm{~h}$, was carried out. This included a physical examination, a blood test and the informant and participant interviews. Ethical approval was sought and received from the King's College London ethics committee and by local ethics committees.

\section{Interviews and measurements}

The interviews, translated into the different languages, lasted around $2-3 \mathrm{~h}$ and collected data necessary to: mental disorders diagnoses (including dementia, depression and anxiety), measurement of comorbid physical illnesses, sociodemographics, risk factors 
specific to dementia and chronic diseases, health-service usage, disability, care arrangements and caregiver strain. ${ }^{11}$ All the scales were translated, back-translated and tested for conceptual uniformity and acceptability, and finally reviewed by local informants.

\section{Sociodemographics}

Age was assessed from formal documents, informant report and, in cases of incongruity, an event calendar. The marital status and gender of the participants, together with the complete list of coresidents, including ages and relationship, were also collected. Socioeconomic status was recorded by measuring a number of variables, including level of education (none/minimal/completed primary/completed secondary/tertiary), income and sources of income, occupational status and food insecurity. Place of birth and residence during adult and later years were also recorded.

\section{Mental health}

Anxiety was measured by using the Geriatric Mental State Examination (GMS) and its associated diagnostic algorithm: the Automated Geriatric Examination for Computer Assisted Taxonomy (AGECAT). ${ }^{12,13}$ The AGECAT algorithm produces a computerised psychiatric diagnosis from data collected in the full GMS interview. All the symptom components are grouped into one of eight diagnostic 'clusters', with level of diagnostic confidence of $0-5$ allocated to the subject of each cluster. The clusters are: organic brain syndrome (dementia); schizophrenia; mania; neurotic and psychotic depression; and obsessional, hypochondriac, phobic, and anxiety neuroses. Scores of 3-5 denote probable cases, 1 and 2 denote subcases, and 0 denotes no or negligible relevant symptoms. Agreement between psychiatrists and AGECAT in making diagnostic decisions has been shown to be high in several studies on different populations. ${ }^{12,13}$ The focus for the GMS, as its name implies, is 'state', specifically, symptoms present in the previous 1 month, and although the GMS does not map precisely onto the DSM or ICD categories, it was calibrated for the detection of disorders of clinical severity, and it has been used in several studies ${ }^{9,14,15}$ to describe generalised anxiety disorder.

The prevalence of anxiety was defined as the frequency of existing cases of anxiety in the given population. Those having a score of at least three in the GMS/AGECAT Stage I anxiety cluster, which is intended to correspond to clinical levels of anxiety, ${ }^{12}$ were considered as 'cases'. Subthreshold disorders (e.g. scores of one and two in the GMS/AGECAT Stage I anxiety cluster), which did not reach clinical levels, were also analysed and reported. Dementia was measured by using the $10 / 66$ algorithm. ${ }^{16}$ The ICD- $10^{17}$ criteria derived from a computerised algorithm applied to the GMS were used to categorise participants with depression.

\section{Other measures}

Activity limitation and participation restriction was estimated by using the World Health Organization Disability Assessment Schedule II (WHODAS-II), ${ }^{18}$ developed by WHO as a culture-fair assessment to use in cross-cultural studies. We also measured whether the informant perceived any anxiety in the participants and the level of distress that this caused. This was done by including the Neuropsychiatric Inventory Questionnaire (NPI) ${ }^{19}$ questions: 'Does your ( $\mathrm{xxxx}$ ) become upset when separated from you? Does he or she have any other signs of nervousness, such as shortness of breath, sighing, being unable to relax, or feeling excessively tense?. The distress level that was experienced by the informant was rated as no distress, minimal, mild, moderate, severe and extreme. The prevalence of perceived anxiety was reported and correlated with the GMS/AGECAT diagnoses.

\section{Statistical analyses}

Version 2.1 of the 10/66 data archive was used for all the analyses, which were carried out in STATA (version 10.1) for Windows. The prevalence of GMS/AGECAT Stage I anxiety syndrome was described and stratified by age and gender and robust $95 \%$ confidence intervals adjusted for household clustering were reported. The standardised prevalence was also reported, to allow comparisons to be made across the study sites, after adjustment for the compositional effects of age, gender and education.

We modelled potential risk factors by using Poisson regression models, which were fitted for each country and provided mutually adjusted prevalence ratios. A fixed-effects meta-analysis was used to combine the countries' prevalence ratios, and an estimate of heterogeneity was provided by the Higgins $I^{2}{ }^{20}$

Level of agreement between the GMS/AGECAT diagnoses and the informant reports were measured by kappa statistics and reported with their 95\% CIs. Spearman's rank correlations of NPI severity scores on the anxiety item and the AGECAT anxiety scores were also calculated.

Finally we investigated whether anxiety had an impact on disability by analysing the distribution of mean WHODAS disability scores, which were reported with their standard deviations. Having 15 days or more of disability in the previous month was defined as severe disability. We then ran Poisson regression models, adjusted for age, gender, 10/66 dementia and number of comorbid illnesses in participants with anxiety and subthreshold anxiety, setting the reference as being free from anxiety. This analysis was also adjusted for ICD-10 depression to see whether there was an independent effect of anxiety on disability scores. Population-attributable prevalence fractions were calculated within the Poisson regression framework by using the Stata aflogit command, which also allows adjustment for confounders. Population-attributable prevalence fractions provide the proportion of existing severe disability that might be averted if that exposure was avoided.

\section{Results}

\section{Sample characteristics}

A total of 15021 interviews were carried out. In all sites, women outnumbered men (62\% women) (online Table DS1). A higher proportion of older people was recorded in South America (in particular Cuba, Dominican Republic, Peru and Mexico), a sign that demographic ageing is more advanced. Higher levels of completed education were registered in the urban areas of all centres, whereas minimal or low education was common in rural areas. Food insecurities were relatively common in India $(14.1 \%$ in rural v. $20.8 \%$ in urban India), rural Peru (13.5\%) and Dominican Republic (12.1\%). The number of missing values was very low (online Table DS1).

\section{Prevalence of anxiety}

The prevalence of anxiety estimated by Stage I GMS/AGECAT diagnoses is shown in Fig. 1. The crude prevalence ranged from 0.2 to $8.9 \%$, where China had the lowest, and Dominican Republic the highest estimates. Standardisation did not affect the figures radically: rural China remained the area with the lowest prevalence $(0.1 \%)$, whereas urban Peru had the highest with 9.6\%. Women had a higher prevalence of anxiety (both clinical and subclinical 
levels) in all sites (online Table DS2). The prevalence estimate of comorbid anxiety and depression ranged from $0.3 \%$ (rural India) to $4.5 \%$ (urban Peru); we exclude China as the number of cases was negligible (Fig. 1). Comorbid depression in participants with anxiety was quite high, and reached almost $50 \%$ in Dominican Republic (online Table DS2). In all centres more than one-fifth of anxiety was comorbid with depression. The distribution of anxiety symptoms that did not reach clinical significance was also measured (online Table DS3). Most centres, with the exception of China and rural India, had a prevalence of subthreshold anxiety well above the $30 \%$ mark, and in a number of sites this estimate was closer to almost one-half of the population. The severity of anxiety in the Latin centres was also higher. In total 235 cases of severe anxiety (2.3\% prevalence), corresponding to level 4 of the GMS/AGECAT output, and 110 cases of very severe anxiety (1.0\% prevalence), equivalent to level 5 of the GMS/AGECAT schedule, were found within Latin America. In India on the other hand the prevalence of severe anxiety was less than half $(0.7 \%)$ and the estimate for very severe anxiety was only $0.2 \%$.

\section{Factors associated with anxiety}

Factors associated with anxiety were investigated in each country and meta-analysed estimates are found in Table 1. We could not estimate prevalence ratios for the Chinese centres, as too few cases occurred. For this reason, the data from China were not included in other analyses.

Similar patterns were found across the sites, although the magnitude of associations changed. Younger age, female gender and lower education were all associated with higher anxiety prevalence. The association between female gender and anxiety was strongest in Cuba and India (prevalence ratio $(P R)=2.0$ ), whereas the inverse relationship between anxiety and age was primarily found in the Cuban and Venezuelan sites.

The factors that had stronger associations with anxiety after pooled estimations were food insecurity $\mathrm{PR}=1.8$ (95\% CI 1.4-2.1), increasing number of physical impairments $\mathrm{PR}=2.3(95 \% \mathrm{CI}$
2.1-2.6) and having a diagnosis of dementia $\mathrm{PR}=1.5$ (95\% CI 1.2-1.8). Living in an urban environment, as measured in Peru, Mexico and India, was associated with the highest prevalence: $\mathrm{PR}=3.3$ (95\% CI 2.0-5.0).

The protective effect of rural environments was explored in more depth in Table 2. We estimated the prevalence of anxiety and calculated adjusted prevalence ratios in participants living in urban settings when the surveys were conducted, but who had lived in rural environments during their lives. The prevalence of anxiety was inversely linked to the length of residence in rural settings (Table 2). However, after adjustment for potential confounders this trend was not found to be statistically significant.

\section{Recognition of anxiety by informants}

When we asked the informants to assess whether the participants suffered from anxiety (NPI), we found a two- to threefold increase in the prevalence of anxiety compared with the estimates obtained by using the GMS/AGECAT system (Table 3). A total of $40 \%$ of the informants were children of the participants, $30 \%$ spouses, $8 \%$ sons- or daughters-in-law, $4 \%$ siblings and the rest included other relatives, friends and neighbours. Anxiety was identified by the informants in less than $40 \%$ of GMS anxiety cases, with the lowest prevalence of $22.2 \%$ in India and the highest of $38.5 \%$ in Cuba. Between $6.4 \%$ (India) and $18.2 \%$ (Cuba) of the informants thought that the participants suffered from anxiety when not confirmed by the GMS/AGECAT diagnosis. We therefore checked whether these informants were recognising different symptoms, such as depression or subclinical anxiety. More than one-half of those who did not have a GMS/AGECAT anxiety diagnosis had either subclinical levels of anxiety (36.9\%) or ICD-10 depression (15.1\%). Kappa values showed very slight agreements between the two different measurements (Table 3), but greater than predicted by chance alone in all the centres. These values did not significantly change when participants with subclinical level of anxiety were included (data not shown).

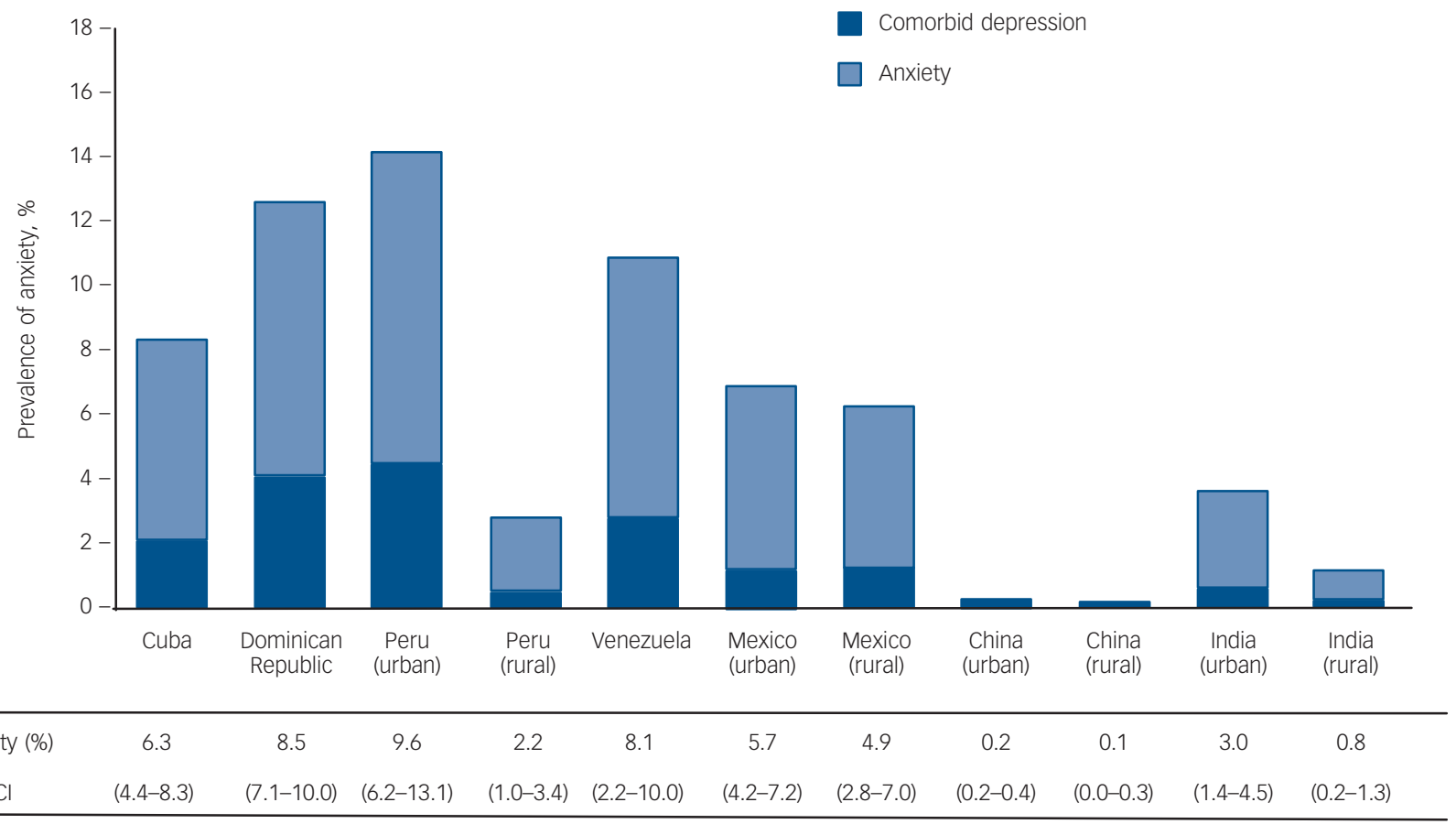

Fig. 1 Age-, gender- and education-standardised prevalence $(95 \% \mathrm{Cl})$ of Geriatric Mental State Examination/Automated Geriatric Examination for Computer Assisted Taxonomy (GMS/AGECAT) Stage I anxiety, and percentage of comorbid ICD-10 depression. 


\begin{tabular}{|c|c|c|c|c|c|c|c|c|}
\hline & $\begin{array}{l}\text { Age (5-year } \\
\text { groups) }\end{array}$ & $\begin{array}{l}\text { Male } \\
\text { gender }\end{array}$ & Education & $\begin{array}{l}\text { Married or } \\
\text { cohabiting }\end{array}$ & $\begin{array}{l}\text { Food } \\
\text { insecurity }\end{array}$ & $\begin{array}{l}\text { Physical } \\
\text { illnesses, } n\end{array}$ & $\begin{array}{l}\text { Dementia } \\
\text { diagnosis }\end{array}$ & $\begin{array}{l}\text { Living in a } \\
\text { rural area }\end{array}$ \\
\hline \multicolumn{9}{|l|}{ Location, PR (95\% Cl) } \\
\hline Cuba & $0.7(0.6-0.8)$ & $0.3(0.2-0.5)$ & $0.9(0.7-1.1)$ & $0.9(0.8-1.1)$ & $1.4(0.8-2.5)$ & $2.1(1.6-2.8)$ & $1.1(0.6-1.9)$ & $n / a$ \\
\hline Dominican Republic & $0.9(0.8-1.0)$ & $0.8(0.6-1.2)$ & $1.0(0.6-1.2)$ & $0.9(0.8-1.1)$ & $2.1(1.5-2.7)$ & $2.9(2.7-3.6)$ & $1.3(0.9-1.9)$ & $n / a$ \\
\hline Peru & $0.9(0.7-1.0)$ & $0.5(0.3-0.8)$ & $0.9(0.8-1.1)$ & $1.0(0.8-1.1)$ & $2.1(1.3-3.4)$ & $2.4(2.0-3.1)$ & $1.7(1.0-2.7)$ & $0.3(0.2-0.6)$ \\
\hline Venezuela & $0.8(0.7-0.9)$ & $0.7(0.4-1.0)$ & $1.0(0.8-1.2)$ & $0.9(0.8-1.1)$ & $1.7(1.1-2.7)$ & $3.4(2.6-4.5)$ & $3.0(1.9-4.7)$ & $\mathrm{n} / \mathrm{a}$ \\
\hline Mexico & $0.8(0.7-1.0)$ & $0.5(0.3-0.8)$ & $0.9(0.7-1.1)$ & $1.1(0.9-1.1)$ & $1.4(0.7-2.7)$ & $2.2(1.6-2.9)$ & $1.6(1.0-2.7)$ & $0.6(0.4-1.0)$ \\
\hline India & $1.0(0.8-1.4)$ & $0.3(0.1-0.6)$ & $0.9(0.6-1.4)$ & $1.1(0.8-1.5)$ & $1.7(0.9-3.1)$ & $1.3(0.8-2.2)$ & $2.5(1.3-4.6)$ & $0.2(0.2-0.5)$ \\
\hline China $^{a}$ & - & - & - & - & - & - & - & - \\
\hline $\begin{array}{l}\text { Meta-analysed estimate, } \\
\text { PR (95\% Cl) }\end{array}$ & $0.8(0.8-0.9)$ & $0.5(0.4-0.6)$ & $0.9(0.8-1.0)$ & $1.0(0.9-1.1)$ & $1.8(1.4-2.1)$ & $2.3(2.1-2.6)$ & $1.5(1.2-1.8)$ & $0.3(0.2-0.5)$ \\
\hline$\chi^{2}$ test for heterogeneity $(P)$ & $12.7(0.03)$ & $14.3(0.01)$ & $1.06(0.96)$ & $3.9(0.56)$ & $2.4(0.80)$ & $16.7(0.006)$ & $8.1(0.15)$ & $4.3(0.12)$ \\
\hline Higgins $I^{2}, \%$ & 60.6 & 65.1 & 0.0 & 0.0 & 0.0 & 69.1 & 38.0 & 53.8 \\
\hline
\end{tabular}

Table 2 The relationship between anxiety and migrations from rural to urban environments

\begin{tabular}{|c|c|c|c|c|}
\hline & \multicolumn{4}{|c|}{ Participants in urban environment at time of survey } \\
\hline & $\begin{array}{l}\text { Never } \\
\text { migrated }\end{array}$ & $\begin{array}{l}\text { Born in rural } \\
\text { environment }\end{array}$ & $\begin{array}{l}\text { Resident in rural } \\
\text { environment at age } 20-60\end{array}$ & $\begin{array}{l}\text { Resident in rural } \\
\text { environment at age } 60\end{array}$ \\
\hline Participants, $n$ & 7893 & 1880 & 386 & 73 \\
\hline Anxiety prevalence ratios $(95 \% \mathrm{Cl})$ & $6.5(6.0-7.0)$ & $6.3(5.3-7.5)$ & $5.7(3.6-8.5)$ & $5.5(1.5-13.4)$ \\
\hline Adjusted prevalence ratios ${ }^{\mathrm{a}}(95 \% \mathrm{Cl})$ & 1.0 (Reference) & $0.9(0.8-1.0)$ & $0.7(0.4-1.1)$ & $1.3(0.5-3.1)$ \\
\hline
\end{tabular}

\begin{tabular}{|c|c|c|c|c|c|}
\hline & $\begin{array}{l}\text { GMS }^{\text {a }} \text { prevalence } \\
\quad(95 \% \mathrm{Cl})\end{array}$ & $\begin{array}{l}\mathrm{NPI}^{\mathrm{b}} \text { prevalence } \\
(95 \% \mathrm{Cl})\end{array}$ & $\begin{array}{l}\text { GMS anxiety identified by } \\
\text { the informant, } \%(n / N)\end{array}$ & $\begin{array}{l}\text { Anxiety identified by informant } \\
\text { not confirmed by GMS, \% }(n / N)\end{array}$ & $\begin{array}{c}\text { Kappa agreement } \\
(95 \% \mathrm{Cl})\end{array}$ \\
\hline Cuba & $4.2(3.5-4.9)$ & $19.1(17.7-20.5)$ & 38.5 (47/122) & $18.2(509 / 2793)$ & $0.08(0.04-0.11)$ \\
\hline Dominican Republic & $8.7(7.4-9.9)$ & $18.2(16.5-19.9)$ & $30.2(54 / 179)$ & $17.3(316 / 1829)$ & $0.09(0.04-0.13)$ \\
\hline Peru & $7.0(5.9-8.2)$ & $14.0(12.5-15.6)$ & $24.3(33 / 136)$ & $13.3(238 / 1795)$ & $0.08(0.03-0.13)$ \\
\hline Venezuela & $7.7(6.5-8.9)$ & $12.7(11.2-14.2)$ & $24.5(40 / 157)$ & $12.1(215 / 1782)$ & $0.10(0.05-0.16)$ \\
\hline Mexico & $5.1(4.2-6.2)$ & $17.4(15.0-19.1)$ & $34.6(36 / 104)$ & $16.5(313 / 1899)$ & $0.09(0.04-0.13)$ \\
\hline India & $2.3(1.7-2.9)$ & $6.7(5.7-7.9)$ & $22.2(10 / 45)$ & $6.4(125 / 1953)$ & $0.08(0.02-0.14)$ \\
\hline
\end{tabular}

Subjective distress caused to the informant by the participants' anxiety was also measured. As expected, the informants of those who had a confirmed GMS/AGECAT diagnosis reported the distress to be significantly higher than those where the GMS anxiety symptoms did not reach clinical levels $(P$-value for the paired $t$-test was $0.026(t=3.13$, d.f. $=5))$. Spearman rank correlations of NPI severity scores and AGECAT anxiety scores showed slight to fair correlations (range of Spearman's rho $(\rho)=0.13-0.22$ ).

\section{The impact of anxiety on disability}

In order to investigate the effect of anxiety on disability we reported the distribution of mean WHODAS disability scores in participants with anxiety, subthreshold anxiety and those without symptoms (Table 4). Anxiety symptoms that did not reach diagnostic levels had intermediate scores across the sites, whereas the means for participants with anxiety were almost double compared with those without anxiety. This was confirmed by running Poisson regressions across the sites. All the prevalence ratios were adjusted for age, gender, 10/66 dementia, number of physical illnesses and ICD-10 depression. Having an anxiety syndrome was significantly associated with severe disability in all centres with the exception of Peru. Subthreshold anxiety was also associated with severe disability in all centres. The contribution of anxiety (both threshold and subthreshold) to severe disability was high, as recorded by the population-attributable prevalence fractions: between $2.3 \%$ (Cuba) and $46.8 \%$ (India) of the prevalence of severe disability could be independently attributed to anxiety. Subthreshold anxiety had higher 


\begin{tabular}{|c|c|c|c|c|c|}
\hline & \multirow[b]{2}{*}{$n(\%)$} & \multirow[b]{2}{*}{$\begin{array}{c}\text { WHODAS-II score, } \\
\text { mean (s.d.) }\end{array}$} & \multicolumn{3}{|c|}{ Severe disability ( 15 or more disability days in the past month) } \\
\hline & & & $n / \mathrm{N}(\%)$ & $\begin{array}{l}\text { Adjusted prevalence ratio }{ }^{a} \\
\qquad(95 \% \mathrm{Cl})\end{array}$ & $\begin{array}{l}\text { Population-attributable prevalence } \\
\text { fraction }(95 \% \mathrm{Cl})\end{array}$ \\
\hline \multicolumn{6}{|l|}{ Cuba } \\
\hline No anxiety & $1689(57.4)$ & $9.4(17.3)$ & 221/1664 (13.3) & 1.0 (reference) & - \\
\hline Subthreshold anxiety & $1131(38.4)$ & $18.0(21.9)$ & $256 / 1115(23.0)$ & $1.1(0.9-1.3)$ & $4.5(0.0-9.9)$ \\
\hline Anxiety & $124(4.2)$ & $25.2(22.5)$ & $35 / 124(28.2)$ & $1.1(0.7-1.8)$ & $2.3(1.1-5.5)$ \\
\hline \multicolumn{6}{|l|}{ Dominican Republic } \\
\hline No anxiety & $788(39.2)$ & $8.2(14.4)$ & $64 / 787(8.1)$ & 1.0 (reference) & - \\
\hline Subthreshold anxiety & $1044(51.9)$ & $19.8(20.9)$ & 184/1041 (17.7) & $1.5(1.1-2.0)$ & $18.0(5.3-29.0)$ \\
\hline Anxiety & $179(8.9)$ & $33.2(23.0)$ & 63/178 (35.4) & $2.0(1.2-3.2)$ & $12.0(5.4-18.2)$ \\
\hline \multicolumn{6}{|l|}{ Peru } \\
\hline No anxiety & $941(48.7)$ & $9.7(18.5)$ & 206/742 (27.8) & 1.0 (reference) & - \\
\hline Subthreshold anxiety & $856(44.3)$ & $13.2(18.3)$ & 182/649 (28.0) & $0.9(0.7-1.2)$ & - \\
\hline Anxiety & $136(7.0)$ & $25.2(21.9)$ & 18/98 (18.4) & $0.4(0.2-0.8)$ & - \\
\hline \multicolumn{6}{|l|}{ Venezuela } \\
\hline No anxiety & $878(44.7)$ & $5.1(9.5)$ & 48/782 (6.1) & 1.0 (reference) & - \\
\hline Subthreshold anxiety & $929(47.3)$ & $13.4(17.4)$ & 109/805 (13.5) & $1.9(1.4-2.8)$ & $32.7(19.8-43.5)$ \\
\hline Anxiety & $158(8.0)$ & $26.8(24.3)$ & 26/134 (19.4) & $2.5(1.3-5.0)$ & $12.5(4.2-20.1)$ \\
\hline \multicolumn{6}{|l|}{ Mexico } \\
\hline No anxiety & $1068(53.3)$ & $7.3(14.9)$ & $67 / 1056(6.3)$ & 1.0 (reference) & - \\
\hline Subthreshold anxiety & $831(41.5)$ & $13.4(20.1)$ & 114/821 (13.9) & $1.6(1.2-2.2)$ & $23.8(10.6-35.0)$ \\
\hline Anxiety & $104(5.2)$ & $20.3(24.6)$ & 23/104 (22.1) & $2.5(1.4-4.4)$ & $15.6(7.4-23.1)$ \\
\hline \multicolumn{6}{|l|}{ India } \\
\hline No anxiety & $1171(58.4)$ & $16.3(17.3)$ & $98 / 1027$ (9.5) & 1.0 (reference) & - \\
\hline Subthreshold anxiety & 787 (39.3) & $23.4(20.4)$ & 147/751 (19.6) & $2.9(2.3-3.7)$ & $46.8(39.2-53.4)$ \\
\hline Anxiety & $46(2.3)$ & $29.5(24.6)$ & 16/39 (41.0) & $3.3(1.9-5.8)$ & $33.1(10.0-55.9)$ \\
\hline
\end{tabular}

population-attributable prevalence fractions, given the higher prevalence of subthreshold anxiety in the populations.

\section{Discussion}

\section{Main findings}

This work showed that anxiety is a relatively common disorder among older people, particularly in survey sites in Latin America. The prevalence estimates of anxiety had a similar range to those found in high-income countries, with the exception of China where the prevalence in the older population was negligible. Two previous studies ${ }^{14,15}$ conducted in Europe reported anxiety using the GMS/AGECAT diagnostic system, finding prevalence estimates of $3.2 \%$ and $4.0 \%$ in the Dutch and British populations respectively. This paper showed that India (rural and urban) and rural Peru had slightly lower estimates (between 0.8 and 3.0\%), whereas the other Latin American sites (Cuba, Dominican Republic, urban Peru, Venezuela and Mexico) had a slightly higher prevalence (range: 4.9-9.6\%) than the European studies.

We also found similar anxiety risk factors as those present in the literature, with gender, socioeconomic determinants and comorbid physical illnesses being the most important. Women were reported to have a twofold increase in prevalence of anxiety compared with men. Genetic and biological factors have been proposed to account for this variation, in particular relating to hormones, but also to psychological and social factors. ${ }^{21}$ It is possible that women might experience greater stress, associated with their societal role and that especially in low- and middleincome countries they might have less chance to escape, avoid and modify a stressful environment. Further research that analyses the interaction between social environment and gender in countries with diverse environments (for example, religious, economic, political) is thus needed. There was an inverse relationship between socioeconomic status and the prevalence of anxiety, in particular with education level and food insecurity, in line with the findings in high-income countries. We also found differences between rural and urban areas, with urban areas having a higher prevalence. It is thought that urban environments are more stressful than rural as the rates of crimes, substandard housing, noise pollution, inadequate services and 'social pathologies' are higher in these places. ${ }^{22,23}$ Social support and social interaction are also known to be higher in rural areas, ${ }^{24}$ with denser networks, more often kin based and associated with religious institutions. ${ }^{25}$ However, we cannot exclude the possibility that the differences seen in urban and rural areas are due not to dissimilarities in the settings themselves, but to differences in terms of demographic variables (for example, ethnicity, age and family status) between the people living in those environments. Even after adjustment in the statistical models some residual confounders may still linger. We also found that among the people who lived in urban settings those who had previously lived in rural environments for longer had the lowest prevalence of anxiety, suggesting a cumulative protective effect of the surrounding environment. After adjustment for potential confounders, this relationship changed only for people who had been living in a rural environment at age 60. Although this was not statistically significant, it potentially indicates that moving to a new environment later in life could contribute to increased stress and anxiety symptoms.

We also found a significant association between disability and anxiety. This association was found with subthreshold disorders too, confirming the idea that people with subthreshold syndromes are still affected and impaired, and that if we were to treat and manage subclinical symptoms we could reduce the burden of disability. In this analysis we also adjusted for comorbid 
depression to show that the association between anxiety and disability still holds after accounting for depression, therefore indicating an independent effect of anxiety. The concordance between informant report and anxiety was modest. This suggests that caregivers might have difficulty in recognising the symptoms, therefore hindering a possible route to recognition and treatment.

An interesting result was the low prevalence of anxiety in China. Other studies found extremely low prevalence of mental disorders in China, ${ }^{7,8}$ and it is possible that the stigmatisation of mental illnesses that has been described in this Asian culture ${ }^{26}$ can partially explain this phenomenon. Besides, the recruited participants were elderly people who had experienced the Great Proletarian Cultural Revolution (1966-1976), a period when 'it was considerably ideologically undesirable to be depressed ${ }^{27}$ and when depressive symptoms were denounced as the manifestation of 'magnified individualism that cut people off from the masses' and criticised as absenteeism, indolence and detachment from a more proletarian mindset. ${ }^{28}$ In any event, external signs of psychological distress were culturally unacceptable in the Chinese culture even before the Cultural Revolution, so this theory cannot fully explain our findings. Chuan et $a l^{10}$ found a very low prevalence of GMS anxiety in Singapore, based on a mixed Chinese, Malay and Indian population, suggesting that this phenomenon may be common across Asian countries. Comparison with other countries of similar backgrounds could have helped in identifying and explaining our results, but unfortunately this was not possible in this study. Another issue may be one of cultural idioms for distress, which could be different particularly among older people and those living in rural areas and which have been largely described in the post-Kleinman literature on shenjing shuairuo ('nerve weakness'). ${ }^{26,29,30}$

\section{Strengths and limitations}

A key strength of this study was the ability to compare prevalence of anxiety in low- and middle-income countries using a measurement instrument specifically created for the cross-cultural assessment of mental health in older people. The protocols, the measurement and the diagnosis algorithm used in each centre were the same and this was specifically done to have a fair way of comparing estimates derived from different countries. Having each centres' principal investigators collectively trained and supervised also helped in maintaining a high internal validity within the study. Missing values were not a particular concern in this project, as the number was minimal, and unlikely to have undermined the findings. The only exception was for the severe disability variable; a modest number of missing values were present in the Peruvian centre, which could possibly account for the lower prevalence ratios found.

Some limitations, however, affect this study. The use of catchment areas helped in achieving a high response rate but its drawback is the theoretical loss of generalisability that could have affected our results, as the findings might not be applicable outside these areas and similar districts. It is very difficult to generalise these results to other low- and middle-income countries. Moreover, rural/urban differences could only be explored in three centres.

Although the validation of the GMS/AGECAT diagnostic system for dementia and mood disorders has been substantial, ${ }^{13}$ its validity for anxiety in low- and middle-income countries is somewhat lacking. It would have been appropriate to do a small validation study for the GMS schedule in a subgroup population of the 10/66 sample, to see if good sensitivity and specificity were maintained, but this was not the primary objective of the 10/66 study and it was therefore not carried out. There is no current gold standard for the measurement of anxiety disorders in older people in low- and middle-income countries and even full clinical diagnoses (DSM-IV ${ }^{31}$ and ICD-10) might not fully embrace the cultural aspects and the clinical spectrum of neuroses in later life. Furthermore, in the GMS schedule the 6-month time component (i.e. symptoms most days for at least 6 months), which is required for the clinical diagnosis using the DSM-IV classification, is not present. The GMS version used (B3) also excludes other types of anxiety disorders such as phobias and panic disorders that could have had a different distribution.

Finally, we cannot completely exclude selection bias. It is possible that in some countries the incompatibility of mental disorders and social roles meant that people with anxiety were more likely to have time to take part in the study. Given the very high response rate across the different centres this scenario is unlikely, but it cannot be entirely excluded.

\section{Clinical implications}

The association between disability and the widespread prevalence of late-life anxiety that was found in our sample emphasises the large scale of this problem. However, most of these countries have minimal mental health facilities, do not have specific mental health policies and they do not allocate any specific budget to mental health. ${ }^{32}$ The estimate ${ }^{33}$ that less than $10 \%$ of older adults receive appropriate treatment for anxiety is likely to be lower in those settings.

Psychological therapies, including cognitive-behavioural therapy (CBT) and relaxation training, have been shown to be effective in older populations in systematic reviews. ${ }^{33,34}$ The relative ease with which relaxation training can be delivered, together with its low cost, make it a prime candidate for use in low- and middle-income countries. Psychological therapies might also be preferred to drug treatment, which often has low uptake among elderly people. Fear of addiction, stigma, previous negative experiences and potential drug interactions have all been described to explain older adults' aversion to antidepressants. ${ }^{35}$ Although the majority of risk factors that we found were nonmodifiable, stepped-care prevention approaches have been used successfully in high-income countries. ${ }^{36}$ It is still not known whether these could be effectively translated to other settings but further research will shed light on this.

One of the key steps in treatment provision is the effective recognition of distress. Family and carers share a role in facilitating access to health services by encouraging their distressed contacts to seek help from general practitioners. We were not expecting good agreement between the informant report and the GMS/AGECAT, because anxiety is an internalising disorder and the NPI examines externally observable signs, which would not necessarily match well with a clinical diagnosis. However, lack of symptom recognition highlights the potential role for awareness campaigns that focus on anxiety symptoms. The low agreement could also suggest that very few of the participants were diagnosed with anxiety prior to the study, which could represent lack of services, barriers to access or non-recognition by medical personnel.

This is the first study to assess anxiety and its correlates in older life in different low- and middle-income countries using the same methodology. Prevalence estimates across the sites varied, but were mostly in line with the estimates from highincome countries. As the demographic change will occur more rapidly in these countries, further research into the mental health of older people in low- and middle-income countries is essential, with the inclusion of other specific anxiety disorders. Future research, including the follow-up phase of this study, will give 
more clues on the course and natural history of anxiety. Temporal relationships between the different factors will also be established. The development of new cross-culture assessment tools capable of measuring common mental disorders will help in gaining more accurate estimates, and further research into evidence-based interventions, both of a clinical and public health nature, will drive treatment provision and disorder detection.

A. Matthew Prina MPhil Department of Public Health and Primary Care, Institute of Public Health, University of Cambridge, Cambridge; Cleusa P. Ferri, PhD, Centre for Public Mental Health, Health Service and Population Research Department, Institute of Psychiatry, King's College London, London; Mariella Guerra, MD, Centre for Public Mental Health, Health Service and Population Research Department, Institute of Psychiatry, King's College London, London, UK, Universidad Peruana Cayetano Heredia, Lima and Institute de la Memoria y Desordenes Relacionadas, Lima, Peru; Carol Brayne, MD, FRCP, FFPH, Department of Public Health and Primary Care, Institute of Public Health, University of Cambridge, Cambridge; Martin Prince, MD, MRCPsych, Centre for Public Mental Health, Health Service and Population Research Department, Institute of Psychiatry, King's College London, London, UK

Correspondence: A. Matthew Prina, Department of Public Health and Primary Care, University of Cambridge, University Forvie Site, Robinson Way, Cambridge CB2 OSR, UK. Email: amp68@medschl.cam.ac.uk

First received 21 Jun 2010, final revision 14 Jul 2011, accepted 28 Jul 2011

\section{Funding}

The 10/66 Dementia Research Group's research has been funded by the Wellcome Trust Health Consequences of Population Change Programme (GR066133 - Prevalence phase in Cuba and Brazil; GR08002 - Incidence phase in Peru, Mexico, Argentina, Cuba, Dominican Republic, Venezuela and China), the World Health Organization (India, Dominican Republic, Venezuela and China), the World Health Organization (India,
Dominican Republic and China), the US Alzheimer's Association (IIRG-04-1286 - Peru, Mexico and Argentina), and FONACIT/ CDCH/ UCV (Venezuela). The Rockefeller Foundation supported a dissemination meeting at their Bellagio Centre. Alzheimer's Disease international has provided support for networking and infrastructure.

\section{Acknowledgements}

This is a secondary analysis of data collected by the 10/66 Dementia Research Group (www.alz.co.uk/1066). The 10/66 DRG is led by Martin Prince and coordinated by Cleus Ferri from Institute of Psychiatry, King's College London. The other principal investigators, responsible for research governance in each site are Juan Llibre Rodriguez (Cuba), Daisy Acosta (Dominican Republic), Mariella Guerra (Peru), Aquiles Salas (Venezuela), Ana Luis , KS Jacob (Nellore, India), Joseph D Williams (Chennai, India) and Yueqin Huang (China)

\section{References}

1 Revicki DA, Brandenburg N, Matza L, Hornbrook MC, Feeny D. Health related quality of life and utilities in primary care patients with generalized anxiety disorder. Qual Life Res 2008; 17: 1285-94.

2 Van Hout HPJ, Beekman ATF, de Beurs E, Comijs H, van Marwijk H, de Haan $M$, et al. Anxiety and the risk of death in older men and women. Br J Psychiatry 2004; 185: 399-404.

3 Allgulander C, Lavori PW. Causes of death among 936 elderly patients with 'pure' anxiety neurosis in Stockholm County, Sweden, and in patients with depressive neurosis or both diagnoses. Compr Psychiatry 1993; 34: 299-302.

4 Brenes GA, Guralnik JM, Williamson JD, Fried LP, Simpson C, Simonsick EM, et al. The influence of anxiety on the progression of disability. J Am Geriatr SOC 2005; 53: 34-9.

5 Jorm AF. Does old age reduce the risk of anxiety and depression? A review of epidemiological studies across the adult life span. Psychol Med 2000; 30 $11-22$.

6 Bryant $\mathrm{C}$, Jackson $\mathrm{H}$, Ames $\mathrm{D}$. The prevalence of anxiety in older adults: methodological issues and a review of the literature. J Affect Disord 2008; 109: 233-50.

7 Ma X, Xiang YT, Cai ZJ, Lu JY, Li SR, Xiang YQ, et al. Generalized anxiety disorder in China: prevalence, sociodemographic correlates, comorbidity, and suicide attempts. Perspect Psychiatr Care 2009; 45: 119-27.

8 Yucun S, Shuran L, Changui C, Weixi Z. An epidemiological survey on neuroses of urban elderly in Beijing. Psychiatry Clin Neurosci 1998; 52 (suppl): s288-90.

9 Ghubash R, El-Rufaie O, Zoubeidi T, Al-Shboul QM, Sabri SF. Profile of mental disorders among the elderly United Arab Emirates population: sociodemographic correlates. Int J Geriatr Psychiatry 2004; 19: 334-51.
10 Chuan SK, Kumar R, Matthew N, Kua EH, Ng TP. Subsyndromal depression in old age: clinical significance and impact in a multi-ethnic community sample of elderly Singaporeans. Int Psychogeriatr 2008; 20: 188-200.

11 Prince $M$, Ferri CP, Acosta D, Albanese $E$, Arizaga R, Dewey $M$, et al. The protocols for the 10/66 dementia research group population-based research programme. BMC Public Health 2007; 7: 165-78.

12 Copeland JRM, Dewey ME, Griffiths-Jones HM. A computerized psychiatric diagnosis system and case nomenclature for elderly subjects: GMS and AGECAT. Psychol Med 1986; 16: 89-99.

13 Copeland JR, Prince M, Wilson KCM, Dewey ME, Payne J, Gurland B. The geriatric mental state examination in the 21st century. Int J Geriatr Psychiatry 2002; 17: 729-32.

14 Kvaal K, McDougall FA, Brayne C, Matthews FE, Dewey ME. Co-occurrence of anxiety and depressive disorders in a community sample of older people: results from the MRC CFAS (Medical Research Council Cognitive Function and Ageing Study). Int J Geriatr Psychiatry 2008; 23: 229-37.

15 Schoevers RA, Beekman AT, Deeg DJ, Jonker C, van Tilburg W. Comorbidity and risk-patterns of depression, generalised anxiety disorder and mixed anxiety-depression in later life: results from the AMSTEL study. Int I Geriatr Psychiatry 2003; 18: 994-1001.

16 Prince $M$, Acosta $D$, Chiu $H$, Scazufca $M$, Varghese $M$. Dementia diagnosis in developing countries: a cross-cultural validation study. Lancet 2003; 361 909-17.

17 World Health Organization. The ICD-10 Classification of Mental and Behavioural Disorders: Clinical Descriptions and Diagnostic Guidelines. WHO, 1992.

18 Rehm J, Ustun TB, Saxena S. On the development and psychometric testing of the WHO screening instrument to assess disablement in the general population. Int J Methods Psychiatric Res 2000; 8: 110-22.

19 Cummings JL, Mega M, Gray K, Rosenberg-Thompson S, Carusi DA, Gornbein J. The Neuropsychiatric Inventory: comprehensive assessment of psychopathology in dementia. Neurology 1994; 44: 2308-14.

20 Higgins JPT, Thompson SG. Quantifying heterogeneity in a meta-analysis. Stat Med 2002; 21: 1539-58.

21 World Health Organization. The World Health Report: 2001. Mental Health: New Understanding, New Hope. WHO, 2001.

22 Krupat E, Guild W. Defining the city: the use of objective and subjective measures for community description. J Soc Issues 1980; 36: 9-28.

23 Glass DC, Singer JE. Urban Stress: Experiments on Noise and Social Stressors. Academic Press, 1972.

24 House JS, Umberson D, Landis KR. Structure and processes of social support. Annu Rev Sociol 1988; 14: 293-380.

25 Fischer C. To Dwell among Friends. University of Chicago Press, 1982.

26 Lee S. Diagnosis postponed: shenjing shuairuo and the transformation of psychiatry in post-Mao China. Cult Med Psychiatry 1999; 23: 349-80.

27 Cheung P. Adult psychiatric epidemiology in China in the 80s. Cult Med Psychiatry 1991; 15: 479-96.

28 Wang SG. The politics of private time: changing leisure patterns in urban China. In Urban Spaces in Contemporary China - The Potential for Autonomy and Community in Post-Mao China (eds DS Davis, R Kraus, B Naughton \& EJ Perry). Woodrow Wilson International Center for Scholars, 1995.

29 Kleinman A. Neurasthenia and depression: a study of somatization and culture in China. Cult Med Psychiatry 1982; 6: 117-90.

30 Chang DF, Myers HF, Yeung A, Zhang Y, Zhao J, Yu S. Shenjing shuairuo and the DSM-IV: diagnosis, distress, and disability in a Chinese primary care setting. Transcult Psychiatry 2005; 42: 204-18.

31 American Psychiatric Association. Diagnostic and Statistical Manual of Mental Disorder (4th edn) (DSM-IV). APA, 1994.

32 World Health Organisation. Mental Health Atlas 2005. WHO, 2005.

33 Hendriks GJ, Oude Voshaar RC, Keijsers GPJ, Hoogduin CAL, van Balkom AJLM. Cognitive-behavioural therapy for late-life anxiety disorders: a systematic review and meta analysis. Acta Psychiatr Scand 2008; 117: 403-11.

34 Ayers CR, Sorrel JT, Thorp SR, Loebach Wetherell J. Evidence-based psychological treatments for late-life anxiety. Psychol Aging 2007; 22: 8-17.

35 Givens JL, Datto CJ, Ruckdeschel K, Knott K, Zubritsky C, Oslin DW, et al. Older patients' aversion to antidepressants. A qualitative study. $J$ Intern Med 2006; 21: 146-51.

36 Veer-Tazelaar PJ, van Marwijk HWJ, van Oppen P, van der Horst HE, Smit F, Cuijpers $P$, et al. Prevention of late-life anxiety and depression has sustained effects over 24 months: a pragmatic randomized trial. Am J Geriatr Psychiatry 2011; 19: 230-9. 